\title{
NOTAS SOBRE EL GÉNERO HABENARIA (ORCHIDACEAE) EN MÉXICO
}

\author{
Javier García-Cruz \\ Rolando JimÉnez MACHORRO \\ LUIS SÁNCHEZ SALdAÑa \\ Herbario AMO \\ Apdo. postal 53-123 \\ 11520 México, D.F. \\ email: eric@intemet.com.mx \\ Adolfo Espejo Serna \\ Y \\ Ana Rosa López-Ferrari \\ Herbario Metropolitano \\ Departamento de Biología \\ División de Ciencias Biológicas y de la Salud \\ Universidad Autónoma Metropolitana-Iztapalapa \\ 09340 México, D.F. \\ email: aes@xanum.uam.mx
}

\section{RESUMEN}

Se neotipifica Orchis entomantha Lex. (=Habenaria entomantha (Lex.) Lindl.), se lectotipifica $H$. acutiflora A. Rich. \& Galeotti que se considera sinónimo de $H$. entomantha y se describen como nuevas para la ciencia Habenaria rosulifolia Espejo \& López-Ferrari y H. gonzaleztamayoi García-Cruz, R. Jiménez \& L. Sánchez, ambas procedentes del estado de Morelos.

\section{ABSTRACT}

Orchis entomantha Lex. (=Habenaria entomantha (Lex.) Lindl.) is neotypified, $H$. acutiflora A. Rich. \& Galeotti is lectotypified and considered as synonym of $H$. entomantha. Habenaria rosulifolia Espejo \& López-Ferrari and H. gonzaleztamayoi García-Cruz, R. Jiménez \& L. Sánchez, both from the state of Morelos, are described as new species.

El género Habenaria Willd. (s. I.) comprende alrededor de 600 taxa de distribución cosmopolita y sin duda alguna constituye uno de los géneros de orquídeas terrestres con más problemas taxonómicos y nomenclaturales, debido al gran número de componentes 
taxa que lo constituye y a que presenta varios complejos de especies cuya delimitación es difícil (González-Tamayo, 1993), particularmente cuando se trabaja con material herborizado, ya que gran parte de los caracteres diagnósticos se encuentran en las flores y éstos no son fáciles de observar en ejemplares prensados. Lo anterior se ve reflejado en el altísimo número de nombres publicados para el género. El Index Kewensis incluye 1762 en tanto que en el Gray Card Index se registran 367 epítetos para el mismo. Sin embargo y a pesar de lo anterior, existen todavía varias especies no descritas.

La última revisión del género para Norteamérica (Ames, 1910) considera 75 especies, pero incluye dentro de Habenaria los géneros Piperia Rydb. y Platanthera Rich., reconocidos actualmente como taxa distintos. Para México, Soto Arenas (1988) registra 27 especies en tanto que Espejo y López-Ferrari (1997) enlistan 41 taxa.

En este panorama destaca un viejo problema nomenclatural que ha sido reconocido por diversos taxónomos (McVaugh, 1985; González Tamayo, 1993) y que es el de Habenaria entomantha (Lex.) Lindl. Un examen, aún incompleto, del material del género depositado en herbarios mexicanos, permite comprobar que el nombre de $\mathrm{H}$. entomantha ha sido tradicionalmente, aunque de manera errónea, aplicado a diversas especies que presentan flores con el perianto verde, el labelo tripartido y los pétalos bipartidos, como es el caso de $H$. guadalajarana S. Watson, $H$. filifera S. Watson, H. jaliscana S. Watson, o a las más recientemente descritas por González Tamayo (1995) H. calicis R. González, H. ortiziana R. González, H. horaliae R. González, H. zamudioana R. González, H. rzedowskii R. González y aún a otras no descritas todavía como $H$. rosulifolia que aquí proponemos.

Orchis entomantha fue descrita por Lexarza (1825) a partir de material proveniente de los alrededores de Valladolid, hoy Morelia y cabe señalar que sólo cuatro de las especies de Habenaria registradas para México fueron descritas antes de $1825(H$. macroceratitis Willd., H. monorrhiza (Sw.) R. Br., H. quinqueseta (Michx.) Sw. y H. repens Nutt.), todas ellas con material original procedente de otros países (Espejo y López-Ferrari, 1997) y ninguna de las cuales corresponde a la especie descrita por Lexarza, por lo que el epíteto entomantha tiene prioridad sobre cualquier otro nombre aplicado a plantas mexicanas.

La descripción original de Orchis entomantha (Lexarza, 1825) en la que se menciona “... perigonium lacteum ..." no deja lugar a dudas de que la especie presenta flores blancas, aunque al final de la descripción se indica que existe una variedad "a." con "... floribus herbaceis ..." a la cual no se le asigna formalmente un nombre y esto, aunado al desconocimiento que se tenía de los taxa del género, seguramente contribuyó a la confusión en la aplicación del nombre. Por otra parte, es bien sabido que el material a partir del cual Lexarza (1825) describió la gran mayoría de sus taxa nuevos, no se conoce y muy probablemente haya desaparecido (Espejo, 1987; Espejo, López Ferrari y Flores, 1993).

Además, a varias especies de flores blancas con pétalos y labelo partidos, se les ha asignado comúnmente el nombre de $H$. clypeata, propuesto por Lindley en 1835 con base en material recolectado por Karwinski probablemente en el estado de Oaxaca.

Por otra parte, en 1845 Richard y Galeotti describieron $H$. acutiflora, también de flores blancas, basándose en material recolectado por el último autor en los alrededores de Jesús del Monte en el actual municipio de Morelia.

Todo lo anterior ha provocado una situación nomenclatural confusa que necesita ser resuelta. Por esta razón y con la idea de solucionar el problema, visitamos los alrededores 
de Morelia, la localidad tipo de Orchis entomantha señalada en el protólogo, para recolectar material que nos permitiera definir cuál es en realidad la especie descrita por Martínez de Lexarza. Con base en la revisión cuidadosa de los ejemplares recolectados, así como del material depositado en los herbarios AMES, AMO, EBUM, ENCB, G, IEB, MEXU, P, UAMIZ y W, concluimos que en los alrededores de Morelia crecen varias especies de Habenaria, pero sólo una de ellas presenta flores blancas así como las características morfológicas descritas por Martínez de Lexarza y constituye sin lugar a dudas la especie por él descrita.

Con base en lo anteriormente relatado, proponemos aquí la neotipificación de Orchis entomantha Lex. y la lectotipificación de Habenaria acutiflora A. Rich. \& Galeotti, quedando esta última como sinónimo taxonómico de la primera. A continuación se presentan las descripciones originales de ambos taxa, tal y como aparecen en sus respectivos protólogos (Lexarza, 1825: p. 8; Richard y Galeotti, 1845: p. 29)

\section{ORCHIS ENTOMANTHA}

O. Labello biglanduloso quinque partito; laciniis linearibus revolutis; calcare ovario longiore; foliis vaginantibus ovatis, quinque nerviis.

Planta terrestris, vix pedalis.--Bulbus solidus, subglobosus indivisus; radiculae filiformes.--Caulis simplex, angulatus, erectus.--Folia ovata, rugata, alterna, vaginantia, nerviis quinque longitudinalibus.--Spica florum laxa. Flores alterni, bracteis lanceolatis, carinatis, involuti.--Perigonium lacteum, concavum, 5 fidum; tribus segmentis superioribus connatis; duobus lateralibus divergentibus acutis.--Labellum quinque-partitum, concolor, laciniis lineari-setaceis revolutis.--Gynostemum capitatum, conico ovatum, obscure bifidum. Anthera bilocularis supra excavata. Pollinis massulae duae obovatae, pedicellatae, lutescentes. Stigma cavum, intra duos loculos antherae hians.--Ovarium triquetrum nonnihil contortum. Calcar sive Nectarium longissimum, filiforme, tubulatum, intra labellum et gynotesmum (sic) excavatum, duabus glandulis virescentibus versus faucem comiculi prominentibus.--Capsula ut in congeneribus.

Habitat prope Vallisoletum; floretque Julio et Augusto. Obs. Orchidi 5 setae Michauxii planta parum affinis; characteribus plurimis sane diversa. $L$.

a. Orchis entomantha, floribus herbaceis. -- Prope Vallisoletum.

99. H. acutiflora Nob. Foliis ovali-oblongis, strictis; flor. parvulis albidis, sepalo supremo lanceolato, acutissimo, dorso glanduloso subcristato: labelli laciniis lateral linearibus longioribus, intermedia linguaeformi lanceolata.

Habenaria entomantha (Lex.) Lindl., Gen. Sp. Orchid. pl. 311. 1835. Basónimo: Orchis entomantha Lex., in La Llave \& Lex., Nov. Veg. Descr. 2: Orchid. Opusc. 8. 1825. Tipo: México, Michoacán, Habitat prope Vallisoletum, J. Martínez de Lexarza (No localizado). Neotipo [aquí designado]: México, Michoacán, municipio de Acuitzio del Canje, $7 \mathrm{~km}$ al S de Acuitzio del Canje, rumbo a Villa Madero, bosque de pino-encino, lugares abiertos, ca. 
1900 m s.n.m., 28.VIII.1986, A. Espejo, M. A. Soto Arenas e I. Ávila 2709 (UAMIZ 17309!; IEB!). Fig. 1.

H. acutiflora A. Rich. \& Galeotti, Ann. Sci. Nat. Bot. ser. 3. 3: 29. 1845. Tipo: México, Michoacán, municipio de Morelia, Jesús del Monte, 7000 ft., fl. blanches, XI-IV.1840, H. Galeotti 5212 (lectotipo [aquí designado]: P!).

Hierbas terrestres, perennes, erectas, de 16 a $35 \mathrm{~cm}$ de alto. Tuberoide elipsoide, de 1 a $2 \mathrm{~cm}$ de largo por 6 a $15 \mathrm{~mm}$ de diámetro. Raíces delgadas, pubescentes, fasciculadas. Hojas 6 a 10, angostamente elípticas a lanceoladas, de 1.5 a $8 \mathrm{~cm}$ de largo por 6 a $11 \mathrm{~mm}$ de ancho. Inflorescencia racemosa, de 3 a $9 \mathrm{~cm}$ de largo, con 4 a 12 flores. Brácteas florales conduplicadas, lanceoladas, acuminadas, de 1.5 a $3.5 \mathrm{~cm}$ de largo por 4 a $7 \mathrm{~mm}$ de ancho, generalmente más largas que el ovario, 5-nervadas. Flores ascendentes, de 1.7 a $2.3 \mathrm{~cm}$ de diámetro, simultáneas, blancas. Ovario erecto, curvado hacia el ápice, glabro, de 1.3 a $1.7 \mathrm{~cm}$ de largo. Sépalo dorsal erecto, cóncavo, ovado a elíptico, subagudo a obtuso, 3-nervado, con una quilla diminutamente papilosa en el dorso, de 8 a $11 \mathrm{~mm}$ de largo por ca. $6 \mathrm{~mm}$ de ancho. Sépalos laterales oblicuamente lanceolados con una quilla papilosa en el dorso, agudos, 3-nervados, de 9 a $12 \mathrm{~mm}$ de largo por 4 a $5 \mathrm{~mm}$ de ancho. Pétalos bilobados, el lóbulo posterior en la misma posición que el sépalo dorsal y adnado a éste, oblicuamente oblongo-oblanceolado, falcado, agudo, 2-nervado, de 9 a 12 mm de largo por 2 a $3 \mathrm{~mm}$ de ancho; el lóbulo anterior linear-filiforme, incurvado, redondeado en el ápice, 1-nervado, de 11 a $14 \mathrm{~mm}$ de largo por ca. $0.8 \mathrm{~mm}$ de ancho. Labelo trilobado, de 11 a $15 \mathrm{~mm}$ de largo; lóbulo medio oblongo-oblanceolado, agudo, 3nervado, de 8 a $12 \mathrm{~mm}$ de largo por 2 a $3 \mathrm{~mm}$ de ancho; lóbulos laterales divergentes, lineares, redondeados, 1-nervados, de 8 a $14 \mathrm{~mm}$ de largo por $0.8 \mathrm{~mm}$ de ancho, subiguales al lóbulo medio. Nectario dirigido hacia abajo, clavado, dilatado y aplanado en la porción apical, de 18 a $27 \mathrm{~mm}$ de largo por 1.8 a $2 \mathrm{~mm}$ de diámetro. Columna corta, de $6 \mathrm{~mm}$ de largo; procesos estigmáticos con la superficie cóncava, de $2.5 \mathrm{~mm}$ de largo; rostelo carnoso. Antera bilocular, los lóculos formando un ángulo menor de $90^{\circ}$ con respecto a los canales de la antera, de 2 a $3 \mathrm{~mm}$ de largo. Polinario formado por 2 hemipolinarios separados; cada uno con un viscidio esférico y una caudícula filiforme, translúcida; polinios 4, 2 por hemipolinario, obovoides.

Ejemplares examinados: Guerrero, Distrito Mina, Zihuaqueo, Filo Mayor, G. B. Hinton et al. $9295(\mathrm{G})$; El Faisán, E. Langlassé $338(\mathrm{G})$; municipio de Alcozauca, cañada del MiniYao, Rancho, M. C. Rojas 11 (UAMIZ); Jalisco, municipio de Mascota, 9 km después de Mascota, rumbo a Ameca, A. Espejo y A. R. López-Ferrari 4189 (UAMIZ); México, municipio de Valle de Bravo, Avándaro, E. Hágsater s. n. (AMO); distrito de Temascaltepec, Rincón del Carmen, G. B. Hinton 1735 (G); distrito de Temascaltepec, Sierrita, G. B. Hinton 4640 (G); municipio de Tejupilco, ca. 2-3 km al NW de Tenería, rumbo a Río Grande, A. R. LópezFerrari, A. Espejo e I. Reyes 2338 (AMO, ENCB, IEB, MEXU, UAMIZ); Michoacán, municipio de Pátzcuaro, Huecorio, J. M. Escobedo 1661 (IEB, UAMIZ); municipio de Acuitzio del Canje, $2 \mathrm{~km}$ al $\mathrm{S}$ de Acuitzio del Canje, rumbo a Villa Madero, A. Espejo, M. A. Soto Arenas e I. Ávila 2717 (UAMIZ); municipio de Chilchota, $1 \mathrm{~km}$ adelante de Rancho Morelos por el camino que va de Carapan a Uruapan, A. Espejo y A. R. López-Ferrari 4203 (UAMIZ); municipio de Uruapan, pedregal, bois Uruapan, H. Galeotti 5218 (W); Michoacán, Cordillera, 
García-Cruz et al.: Notas Sobre el Género Habenaria en México
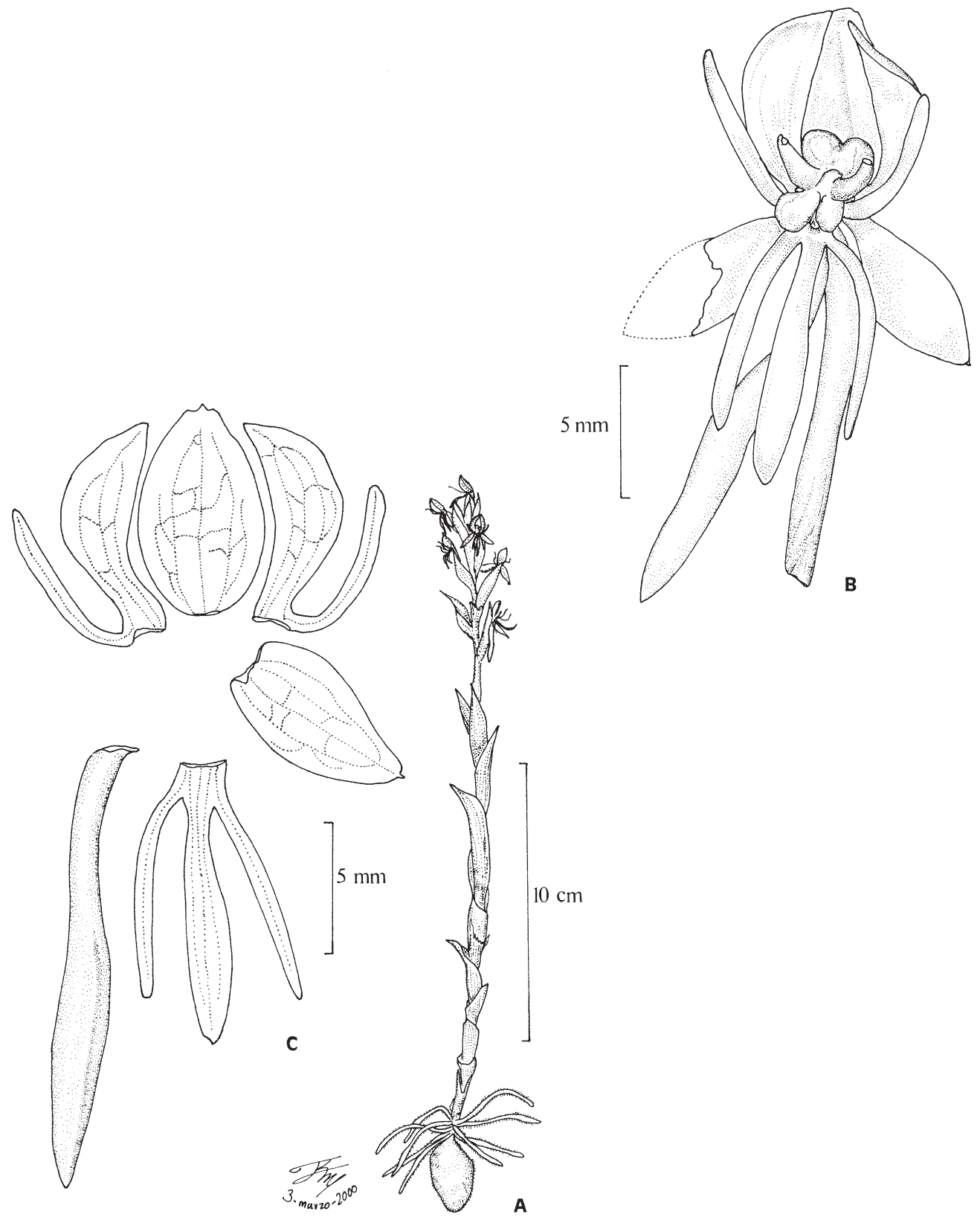

Fig. 1. Habenaria entomantha (Lex.) Lindl. A. hábito; B. flor, vista de tres cuartos; C. flor disecada. 
H. Galeotti $5218(\mathrm{G})$; municipio de Uruapan, Uruapan, H. Galeotti 5219 (G); municipio de Angangueo, Angangueo, in pascuis, K. Hartweg 397 (G); municipio de Morelia, 2 km al noreste del cerro de las Tetillas, V. M. Huerta B. 612 (AMO, UAMIZ); municipio de Huaniqueo, alrededores de Tendeparacua, A. R. López-Ferrari y A. Espejo 2062 (UAMIZ); municipio de Paracho, $5 \mathrm{~km}$ al SW de Paracho, M. Pérez R. 134 (AMO, IEB, UAMIZ); Morelos, municipio de Cuernavaca, lomas al NW de la barranca de La Canoa, al NW de Cuernavaca, A. Espejo, A. R. López-Ferrari, J. García-Cruz, R. Jiménez M. y L. Sánchez S. 5699 (UAMIZ); lomas de Atzingo, E. Lyonnet 500800010 (MEXU); excursión Santa María - Tetela [del Monte], E. Lyonnet 540900037 (MEXU); Tetela del Monte, lomas, E. Lyonnet 550800058 (MEXU); Cuernavaca, O. Nagel sub E. Oestlund 3032 (MEXU); municipio de Huitzilac, alrededores de la laguna Tonatihua, A. Espejo 883 (UAMIZ); laguna Tonatihua, A. Espejo 3317 (UAMIZ); alrededores de la laguna Tonatihua, parque nacional Lagunas de Zempoala, A. R. López-Ferrari, A. Espejo, J. García-Cruz y R. Jiménez M. 2228 (UAMIZ); municipio de Tepoztlán, km 94 del ferrocarril a Cuernavaca, M. Flores C., A. Espejo, A. R. López Ferrari y G. Barroso 119 (UAMIZ); km 64 de la carretera [federal] MéxicoCuernavaca, E. Oestlund 1384 (MEXU); Sin localidad precisa, "Nova, Hispania", M. Sessé y J. M. Mociño 4399 (MA); Oaxaca, Santiago el Grande, W. Karwinski s. n. (G); Sin localidad precisa, K. Hartweg 399 (G).

Como resultado de la exploración intensiva llevada a cabo en el estado de Morelos durante los últimos tres años, descubrimos dos nuevas especies para la ciencia, que aquí proponemos:

Habenaria rosulifolia Espejo \& López-Ferrari, sp. nov. Fig. 2.

Herba perennis, parva. Inflorescentia pauciflora, flores pro ratione grandes ca. $2 \mathrm{~cm}$ diametro. Folia basalia ovato-elliptica, subrosulata vel rosulata, viridi-grisea, supra iridescentia. Habenariae filferae affinis.

Hierbas terrestres, perennes, erectas, de 9 a $25 \mathrm{~cm}$ de alto. Tuberoide ovoide a elipsoide, arrugado, de 1 a $1.7 \mathrm{~cm}$ de largo por 0.5 a $1.1 \mathrm{~cm}$ de diámetro. Raíces delgadas, pubescentes. Hojas 5 a 7, en ocasiones con una pequeña hoja en la base, elíptica, aguda, revoluta, de 0.6 a $1.5 \mathrm{~cm}$ de largo por 5 a $7 \mathrm{~mm}$ de ancho; seguida por 2 a 3 hojas muy próximas entre sí, las cuales casi forman una roseta, ovado-elípticas, tubulares en la base, imbricadas, obtusas a redondeadas, en ocasiones apiculadas, verde-grisáceas e iridiscentes en el haz, gris-verdosas en el envés, de 1.2 a $3 \mathrm{~cm}$ de largo por 8 a $20 \mathrm{~mm}$ de ancho y con 3 venas resaltadas en el haz; después 2 a 3 hojas similares a las brácteas florales, las inferiores con apariencia similar a las anteriores, las superiores erectas, conduplicadas, lanceoladas y acuminadas, brevemente tubulares en la base, espaciadas, de 1.5 a $3.5 \mathrm{~cm}$ de largo por 5 a $15 \mathrm{~mm}$ de ancho, con una quilla poco prominente en el dorso. Inflorescencia racemosa, de 5 a $12 \mathrm{~cm}$ de largo, el raquis algo sinuoso, ligeramente comprimido y ligeramente aquillado, con 3 a 6 flores. Brácteas florales conduplicadas, lanceoladas, acuminadas, de 1.3 a $3 \mathrm{~cm}$ de largo por 7 a $12 \mathrm{~mm}$ de ancho, 3 a 5-nervadas, ligeramente carinadas y diminutamente papilosas en el dorso, sobrepasando al ovario. Flores ascendentes, de 1.3 a $2 \mathrm{~cm}$ de diámetro, simultáneas, verde-amarillentas. Ovario 


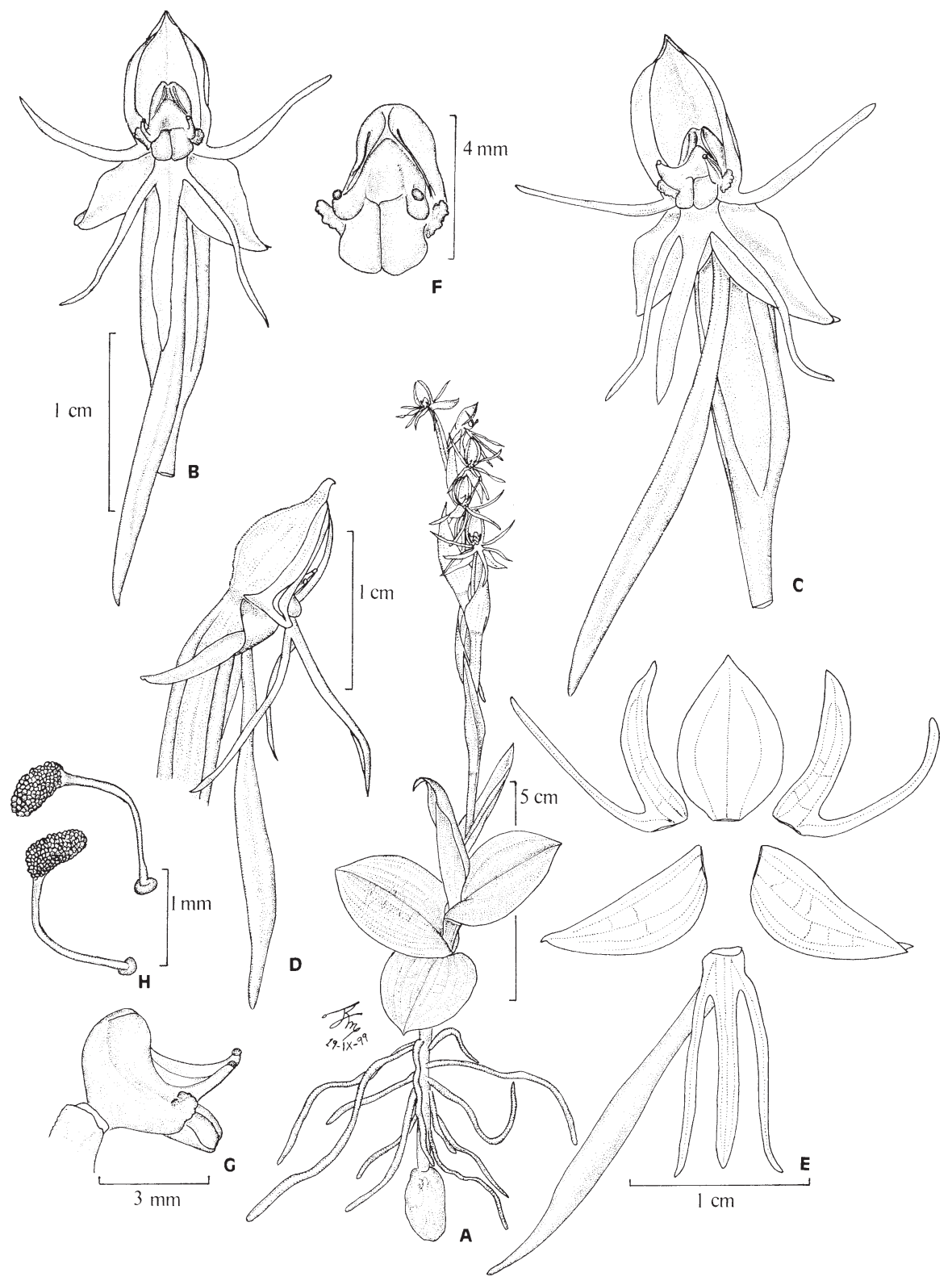

Fig. 2. Habenaria rosulifolia Espejo \& López-Ferrari. A. hábito; B. flor, vista frontal; C. flor, vista de tres cuartos; D. flor, vista lateral; E. flor disecada; F. columna, vista frontal; G. columna, vista lateral; $\mathrm{H}$. hemipolinarios. 
erecto, recto, rollizo, diminutamente papiloso, de 1.5 a $2 \mathrm{~cm}$ de largo. Sépalo dorsal erecto, cóncavo, cuculado, ovado, agudo, ligeramente apiculado, de 6 a $9 \mathrm{~mm}$ de largo por 6 a $8 \mathrm{~mm}$ de ancho, 3-nervado, con una quilla diminutamente papilosa y baja en el dorso. Sépalos laterales reflexos, extendidos, oblicuamente ovado-lanceolados, agudos, mucronados, de ca. $1 \mathrm{~cm}$ de largo por $4 \mathrm{~mm}$ de ancho, 3- a 5-nervados, con una quilla diminutamente papilosa y baja en el dorso. Pétalos extendidos, bilobados, el lóbulo posterior en la misma posición que el sépalo dorsal y adnado a éste, arqueado, erecto, falcado, angostamente lanceolado, agudo, de ca. $9 \mathrm{~mm}$ de largo por ca. $2 \mathrm{~mm}$ de ancho, trinervado; el lóbulo anterior filiforme, redondeado en el ápice, de ca. $9 \mathrm{~mm}$ de largo por ca. $0.6 \mathrm{~mm}$ de ancho, 1-nervado. Labelo trilobado, unguiculado, la uña subcuadrada, de ca. $2 \mathrm{~mm}$ de largo por ca. $2 \mathrm{~mm}$ de ancho; el lóbulo medio linear-oblongo, agudo, de ca. $1 \mathrm{~cm}$ de largo por ca. $1 \mathrm{~mm}$ de ancho, trinervado; los lóbulos laterales divergentes en posición natural, lineares, redondeados en el ápice, de ca. $11 \mathrm{~mm}$ de largo por ca. $0.6 \mathrm{~mm}$ de ancho, uninervados, sobrepasando ligeramente al lóbulo medio. Nectario dirigido hacia abajo, ligeramente arqueado hacia adelante, clavado, dilatado y aplanado en la porción apical, de ca. $2 \mathrm{~cm}$ de largo por $1.6 \mathrm{~mm}$ de diámetro, subtriquetro, más largo que el lóbulo medio del labelo y ligeramente más largo que el ovario, el ápice agudo. Columna de ca. $5.5 \mathrm{~mm}$ de largo por ca. $2.7 \mathrm{~mm}$ de ancho; procesos estigmáticos con la superficie cóncava, formando un ángulo de $90^{\circ}$ con respecto a los canales de la antera, oblongos, de ca. $2.1 \mathrm{~mm}$ de largo por ca. $2.5 \mathrm{~mm}$ de ancho; rostelo carnoso. Antera bilocular, los lóculos divergentes, de $1.8 \mathrm{~mm}$ de largo; los canales cortos, divergentes y ascendentes. Polinario formado por 2 hemipolinarios, de ca. $2.6 \mathrm{~mm}$ de largo, cada uno con un viscidio esférico, sulcado y una caudícula filiforme, translúcida; polinios 4 , obovoides, de ca. $1.3 \mathrm{~mm}$ de largo.

TIPO: México, Morelos, municipio de Huitzilac, cerros alrededor de la laguna Tonatihua, parque nacional Lagunas de Zempoala, 1903'26" N, 99¹9'06" W, bosque de pino-abeto, 2800 m s.n.m., 13.IX.1997, A. R. López-Ferrari, A. Espejo, J. García-Cruz y R. Jiménez M. 2558 (Holotipo: UAMIZ; isotipos: AMES, AMO).

Paratipos: México, Morelos, municipio de Huitzilac, alrededores de la laguna Tonatihua, parque nacional Lagunas de Zempola, 1903'19" N, 99¹8'54" W, 2850 m, bosque de Pinus, en lugares rocosos y asoleados, 7.VIII.1983, A. Espejo 885 (UAMIZ); municipio de Huitzilac, km 13 carretera Tres Marías-Lagunas de Zempoala, parque nacional Lagunas de Zempoala, 1902'12" N, 99¹8'11" W, bosque de pino en ladera rocosa, $2900 \mathrm{~m}$ s.n.m., 13.IX.1997, J. García-Cruz 774 (AMO, IEB, K, MEXU, MO, UAMIZ); municipio de Huitzilac, cerros alrededor de la laguna Tonatihua, parque nacional Lagunas de Zempoala, 1903'28" N, 99¹9'07" W, 2800 m s.n.m., bosque de pino-abeto, 13.IX.1997, J. GarcíaCruz, R. Jiménez M., A. Espejo y A. R. López-Ferrari 777 (AMO, UAMIZ); municipio de Huitzilac, km 13 carretera Tres Marías-Lagunas de Zempoala, parque nacional Lagunas de Zempoala, 1902'12" N, 99¹8'11" W, bosque de pino en ladera rocosa, 2900 m s.n.m., 13.IX.1997, A. R. López-Ferrari, A. Espejo, J. García-Cruz y R. Jiménez M. 2554 (IEB, UAMIZ).

Etimología: El epíteto asignado a este taxon hace referencia a las hojas inferiores arrosetadas. 
Reconocimiento: La nueva especie se reconoce por su pequeño porte, por su inflorescencia pauciflora y con flores relativamente grandes de casi dos centímetros de diámetro y por la presencia de 2 a 3 hojas basales ovado-elípticas, verde-grisáceas e iridiscentes en el haz, que tienden a formar una roseta en la base de la planta. Además, $H$. rosulifolia muestra una clara preferencia por hábitats rocosos por arriba de los $2800 \mathrm{~m}$ s.n.m. dentro de bosques de pino y de pino-abeto. La especie suele encontrarse formando colonias de numerosos individuos.

Hasta el momento esta planta sólo se conoce de dos colonias procedentes del estado de Morelos, en sus límites con el Estado de México, por lo que no sería raro, en el futuro, encontrarla en dicha entidad.

La especie mas parecida al taxon aquí descrito es $H$. filifera S. Watson (1891), a la cual se asemeja en el porte y en el color de las flores. Sin embargo, $H$. rosulifolia presenta las hojas iridiscentes y arrosetadas en la base y una inflorescencia pauciflora con 3 a 6 flores laxamente dispuestas, en tanto que $H$. filifera carece de hojas arrosetadas y la inflorescencia es compacta y lleva generalmente de 9 a 12 flores. Además, en esta última especie las flores son más pequeñas y los lóbulos anteriores de los pétalos y los laterales del labelo, son muy delgados (filíferos) y más largos que el lóbulo posterior y el central respectivamente.

Habenaria gonzaleztamayoi García-Cruz, R. Jiménez \& L. Sánchez, sp. nov. Fig. 3.

Herba perennis, grandis, ca. $50 \mathrm{~cm}$ alta. Inflorescentia longa, floribus lacteo-viridibus. Folia numerosa, discolora, supra atroviridia, subtus pallide viridia.

Hierbas terrestres, perennes, erectas, de ca. $50 \mathrm{~cm}$ de alto. Tuberoide ovoide, arrugado, escasamente piloso, de ca. $2 \mathrm{~cm}$ de largo por $1 \mathrm{~cm}$ de ancho. Raíces delgadas, escasamente pilosas. Hojas ca. 15, en ocasiones la basal sin lámina, las caulinares con la lámina elíptica, tubulares en la base, agudas a mucronadas, enteras, 5- a 7-nervadas, los nervios prominentes en el envés, verdes oscuras por el haz, algo más claras en el envés, glabras, las basales y apicales progresivamente más pequeñas que las medias, de 3 a 7.5 $\mathrm{cm}$ de largo por 0.5 a $2 \mathrm{~cm}$ de ancho. Inflorescencia racemosa, de 7-10 cm de largo, con 12-18 flores. Brácteas florales conduplicadas, lanceoladas, acuminadas, ligeramente carinadas por el dorso en la vena media, aproximadamente tan largas como el ovario, 3nervadas, de 12-15 mm de largo por 5-6 mm de ancho. Flores ascendentes, de ca. $1.2 \mathrm{~cm}$ de diámetro, blanco-verdosas. Ovario curvado, rollizo, liso, de ca. $1.3 \mathrm{~cm}$ de largo. Sépalo dorsal erecto, cóncavo, ovado, agudo, 3-nervado, con una quilla diminutamente papilosa en el dorso, blanco con la vena media verde, de ca. $5 \mathrm{~mm}$ de largo por ca. $4 \mathrm{~mm}$ de ancho. Sépalos laterales algo reflexos, oblicuamente elíptico-lanceolados, agudos, mucronados, con una quilla diminutamente papilosa en el dorso, blancos con la vena media verde, de ca. $7 \mathrm{~mm}$ de largo por ca. $3.5 \mathrm{~mm}$ de ancho. Pétalos bilobados, el lóbulo posterior en la misma posición que el sépalo dorsal y adnado a éste, erecto, falcado, agudo, 3-nervado, blancos hacia la base y ligeramente verdosos hacia el ápice, de ca. $5 \mathrm{~mm}$ de largo por ca. $1.4 \mathrm{~mm}$ de ancho; el lóbulo anterior filiforme, incurvado, redondeado en el ápice, 1nervado, de ca. $5 \mathrm{~mm}$ de largo por ca. $0.5 \mathrm{~mm}$ de ancho. Labelo unguiculado, la uña subcuadrada, de $1 \mathrm{~mm}$ de largo por $1 \mathrm{~mm}$ de ancho, trilobado; el lóbulo medio linear- 


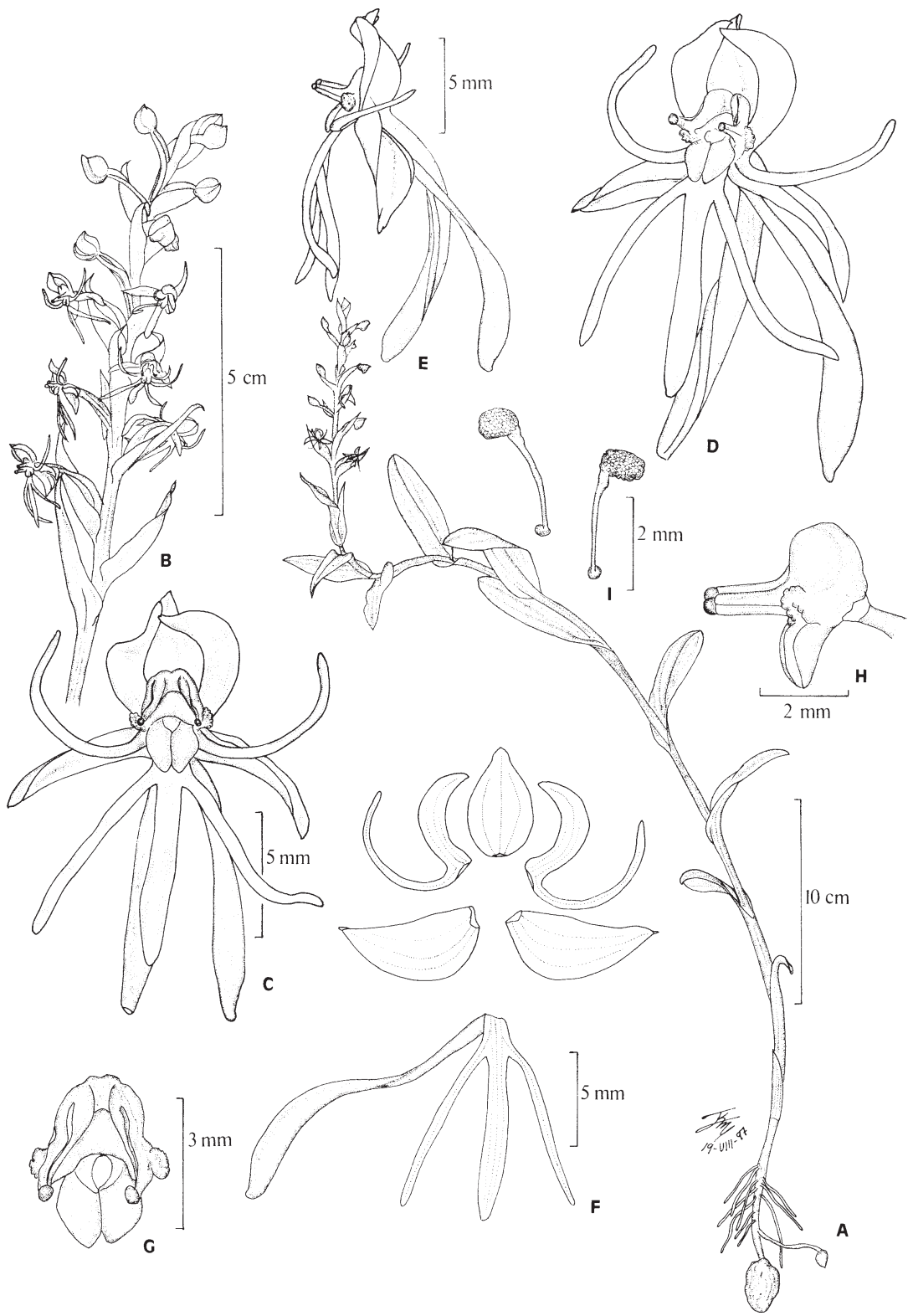

Fig. 3. Habenaria gonzaleztamayoi García-Cruz, R. Jiménez \& L. Sánchez. A. hábito; B. detalle de la inflorescencia; C. flor, vista frontal; D. flor, vista de tres cuartos; E. flor, vista lateral; F. flor disecada; G. columna, vista frontal; H. columna, vista lateral; I. hemipolinarios. 
oblongo, redondeado, blanco hacia la base y ligeramente verdoso hacia el ápice, de 6.5$7 \mathrm{~mm}$ de largo por ca. $1 \mathrm{~mm}$ de ancho; los lóbulos laterales divergentes, filiformes, redondeados, de $8-9 \mathrm{~mm}$ de largo por ca. $0.5 \mathrm{~mm}$ de ancho, sobrepasando ligeramente al lóbulo medio. Nectario dirigido hacia abajo, ligeramente arqueado hacia atrás, clavado, dilatado y aplanado en la mitad apical, de cerca del doble de largo o más que el lóbulo medio del labelo y ligeramente más largo que el ovario, verde, de $1.5 \mathrm{~cm}$ de largo por ca. $1 \mathrm{~mm}$ de diámetro. Columna de ca. $2.5 \mathrm{~mm}$ de largo por ca. $2 \mathrm{~mm}$ de ancho; procesos estigmáticos con la superficie cóncava, de ca. $1.2 \mathrm{~mm}$ de largo, formando un ángulo de $90^{\circ}$ con respecto a los canales de la antera; rostelo carnoso. Antera bilocular, los lóculos paralelos, los canales divergentes y ascendentes, de ca. $1.5 \mathrm{~mm}$ de largo. Polinario formado por 2 hemipolinarios separados; cada uno con viscidio esférico y una caudícula translúcida; polinios obovoides.

TIPO: México, Morelos, municipio de Puente de Ixtla, $12 \mathrm{~km}$ adelante de La Tigra, rumbo a El Zapote, 18²8'14" N, 99²0'47" W, bosque de encino, 1650 m s.n.m., 10.VII.1997, A. Espejo, A. R. López-Ferrari, J. García-Cruz, R. Jiménez M. y L. Sánchez S. 5677 (Holotipo: UAMIZ).

Etimología. Dedicamos esta especie a Roberto González Tamayo, colega del Instituto de Botánica de la Universidad de Guadalajara, especialista en el género Habenaria, quien por mucho tiempo se ha dedicado entusiastamente al estudio de las orquídeas.

Reconocimiento: H. gonzaleztamayoi se reconoce por su porte alto de ca. $50 \mathrm{~cm}$, por su inflorescencia alargada con flores blanco-verdosas y por sus numerosas hojas discoloras, verdes oscuras en el haz y verdes claras en el envés. La especie proviene de bosques de encino más bien secos en ecotonía con bosque tropical caducifolio, a una altitud cercana a los $1650 \mathrm{~m}$. Los individuos crecen aislados y son más bien inconspicuos.

Hasta el momento $H$. gonzaleztamayoi se conoce únicamente de una colección procedente del estado de Morelos, en sus límites con el estado de Guerrero, por lo que podría esperarse que en el futuro, se recolectara en este último.

Habenaria gonzaleztamayoi podría confundirse por sus flores blancas con $H$. emtomantha, sin embargo, ésta última especie tiene un porte más pequeño y hojas mucho mas cortas y menos numerosas. Además las flores de $H$. entomantha son completamente blancas, en tanto que las del taxon aqui propuesto son blanco-verdosas.

\section{AGRADECIMIENTOS}

Deseamos agradecer a Roberto González Tamayo, Ed. Greenwood, Eric Hágsater y Ramón Riba por la cuidadosa revisión del manuscrito, así como por sus sugerencias y observaciones. Los curadores de los herbarios consultados nos otorgaron todas las facilidades para la revisión del material bajo su custodia. En particular queremos expresar nuestra gratitud al Dr. H. J. Tillich del herbario de Munich (M), quien amablemente nos proporcionó fotocopias de los tipos de las especies mexicanas de Habenaria depositados en sus colecciones. Los dibujos fueron elaborados por Rolando Jiménez Machorro. Este 
trabajo fue patrocinado parcialmente por la Comisión Nacional para el Conocimiento y Uso de la Biodiversidad (CONABIO) a través de los proyectos G016, H043 y H098.

\section{LITERATURA CITADA}

Ames, O. 1910. The genus Habenaria in North America. Orchidaceae 4: 1-288.

Espejo, A. 1987. Neotipificación de Cymbidium vexilliferum La Llave \& Lexarza (= Liparis vexillifera) y descripción de Liparis greenwoodiana. Orquídea (Méx.) 10: 365-384.

Espejo, A., A. R. López Ferrari y M. Flores. 1993. Neotipificación de Pitcairnia vallisoletana Lex. (Bromeliaceae) Acta Bot. Mex. 23: 53-58.

Espejo, A. y A. R. López Ferrari. 1997. Las monocotiledóneas mexicanas, una sinopsis florística 1. Lista de referencia. Parte VII. Orchidaceae 1. Consejo Nacional de la Flora de México, A. C., Universidad Autónoma Metropolitana-Iztapalapa y Comisión Nacional para el Conocimiento y Uso de la Biodiversidad, México, D.F. $90 \mathrm{pp}$.

González Tamayo, R. 1992. Dos especies nuevas del género Habenaria (Orchidaceae). Bol. Inst. Bot. Univ. Guadalajara 1: 321-331.

González Tamayo, R. 1993. Algunas consideraciones sobre el género Habenaria (Orchidaceae) en México. Bol. Inst. Bot. Univ. Guadalajara 1: 485-511.

González Tamayo, R. 1995. Algunas especies nuevas de Habenaria (Orchidaceae) con flores verdes en México. Bol. Inst. Bot. Univ. Guadalajara 3: 53-86.

Lexarza, J. M. 1825. In: La Llave, P. y J. M. Lexarza. Novorum vegetabilium descriptiones. Fasciculus 2. Orchidianum opusculum. México. 43 pp.

Lindley, J. 1835. The genera and species of orchidaceous plants. Londres. $553 \mathrm{pp}$.

McVaugh, R. 1985. Orchidaceae In: Anderson W. (ed.). Flora Novo-Galiciana 16. The University of Michigan Press. 363 pp.

Richard, A. y H. Galeotti. 1845. Orchidographie mexicaine, d'après les échantillons, notes et dessins de MM. Galeotti, Linden, Funck, Ghiesbreght. Annales des Sciences Naturelles 3me. Ser. Botanique 3: 15-33.

Soto Arenas, M. A. 1988. Listado actualizado de las orquídeas de México. Orquídea (Méx.) 11: 233272.

Watson, S. 1891. Contributions to American botany. 2. Descriptions of new Mexican species, collected chiefly by Mr. C. G. Pringle in 1889 and 1890. Proc. Amer. Acad. Arts 26: 131-158.

Williams, L. O. 1951. The Orchidaceae of Mexico. Ceiba 2: 1-321. 